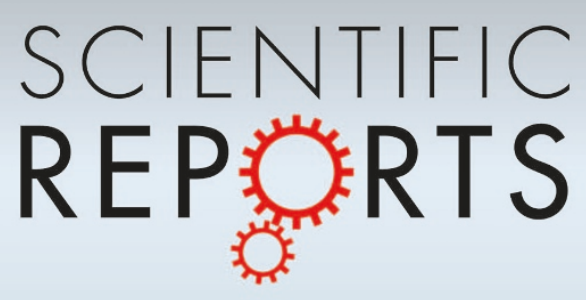

OPEN

SUBJECT AREAS:

SEMICONDUCTORS

ELECTRONIC PROPERTIES AND

MATERIALS

Received

25 September 2014

Accepted

15 January 2015

Published

9 February 2015

Correspondence and requests for materials should be addressed to S.Z. (s.zhou@hzdr.de)

\title{
Hyperdoping silicon with selenium: solid vs. liquid phase epitaxy
}

\author{
Shengqiang Zhou', Fang Liu',2, S. Prucnal' ', Kun Gao',2, M. Khalid', C. Baehtz' , M. Posselt', \\ W. Skorupa' \& M. Helm ${ }^{1,2}$
}

${ }^{1}$ Helmholtz-Zentrum Dresden-Rossendorf, Institute of lon Beam Physics and Materials Research, Bautzner Landstr. 400, 01328
Dresden, Germany, ${ }^{2}$ Technische Universität Dresden, 01062 Dresden, Germany.

\begin{abstract}
Chalcogen-hyperdoped silicon shows potential applications in silicon-based infrared photodetectors and intermediate band solar cells. Due to the low solid solubility limits of chalcogen elements in silicon, these materials were previously realized by femtosecond or nanosecond laser annealing of implanted silicon or bare silicon in certain background gases. The high energy density deposited on the silicon surface leads to a liquid phase and the fast recrystallization velocity allows trapping of chalcogen into the silicon matrix. However, this method encounters the problem of surface segregation. In this paper, we propose a solid phase processing by flash-lamp annealing in the millisecond range, which is in between the conventional rapid thermal annealing and pulsed laser annealing. Flash lamp annealed selenium-implanted silicon shows a substitutional fraction of $\sim 70 \%$ with an implanted concentration up to $2.3 \%$. The resistivity is lower and the carrier mobility is higher than those of nanosecond pulsed laser annealed samples. Our results show that flash-lamp annealing is superior to laser annealing in preventing surface segregation and in allowing scalability.
\end{abstract}

\footnotetext{
C halcogen-hyperdoped silicon (Si) much above the solid solubility limits has been investigated due to its specific physical properties, such as a near-unity broadband (particularly below the Si bandgap) absorption $^{1-4}$, a large enhancement of below-band-gap photocurrent generation ${ }^{5}$, and insulator-to-metal transition $^{6,7}$. These novel properties make chalcogen-hyperdoped Si a promising material for applications in Si-based infrared photodetectors ${ }^{8-10}$ and intermediate band solar cells ${ }^{11-14}$. The insulator-to-metal transition realized in sulfur and selenium doped $\mathrm{Si}$ is driven by deep level impurities, which opens a new material test-bed for insulatorto-metal transition, the long-standing fundamental problem. Note that chalcogen elements in Si generally have much lower solubility limits compared with III and V-column elements. For instance, the solid solubility limit for sulfur in $\mathrm{Si}^{15}$ was determined to be around $10^{16} \mathrm{~cm}^{-3}$. To realize hyperdoping above the solid solubility limits, non-equilibrium methods were used. Femtosecond (fs) or nanosecond (ns) pulsed laser irradiation was successfully applied to prepare chalcogen-hyperdoped Si by melting the Si surface in certain background gases containing chalcogens or with a powder/film of chalcogen on the surface, ${ }^{3,16,17}$. In parallel, by ion implantation and subsequent annealing with ns laser pulses chalcogen-hyperdoped Si have been fabricated ${ }^{1,2,4,18}$. Both $\mathrm{fs}$ and ns (or even longer pulse length) laser annealing (pulsed laser annealing: PLA) at a high enough energy density melts the Si surface ${ }^{19}$ and renders a fast solidification after the laser is off. The solute impurities are trapped at the moving interface when the regrowth time is short enough ${ }^{20-23}$. However, the liquid phase epitaxy associated with fs or ns laser annealing encounters some drawbacks. One is the dopant redistribution and pileup at the surface ${ }^{2,20,24}$. The second is the significant loss of the dopant due to surface evaporation ${ }^{2}$. The third is the formation of polycrystalline materials mainly due to the rejected excess dopants ${ }^{20,25}$. The last but not the least is its missing scalability and reproducibility of PLA, which inhibits the application in microelectronics industry.

In this paper, we propose a novel approach, flash lamp annealing (FLA) in millisecond time duration which allows for a solid phase epitaxy from implanted Si layers, to realize selenium hyperdoped $\mathrm{Si}$. We will show that the solid phase epitaxy also can achieve selenium-hyperdoped Si with larger Hall mobility. Additionally, the problems mentioned above occurring in nanosecond pulsed laser annealing can be solved by flashlamp annealing.
}

\section{Results}

Selenium substitution without diffusion. Figure 1 shows the representative Rutherford backscattering spectrometry (RBS) random and channeling spectra of selenium-implanted Si substrates (Sample SiSe2.3 in Table 1) that were pulsed laser annealed (wavelength: $308 \mathrm{~nm}$, pulse length: $28 \mathrm{ns)}$ or flash lamp annealed 

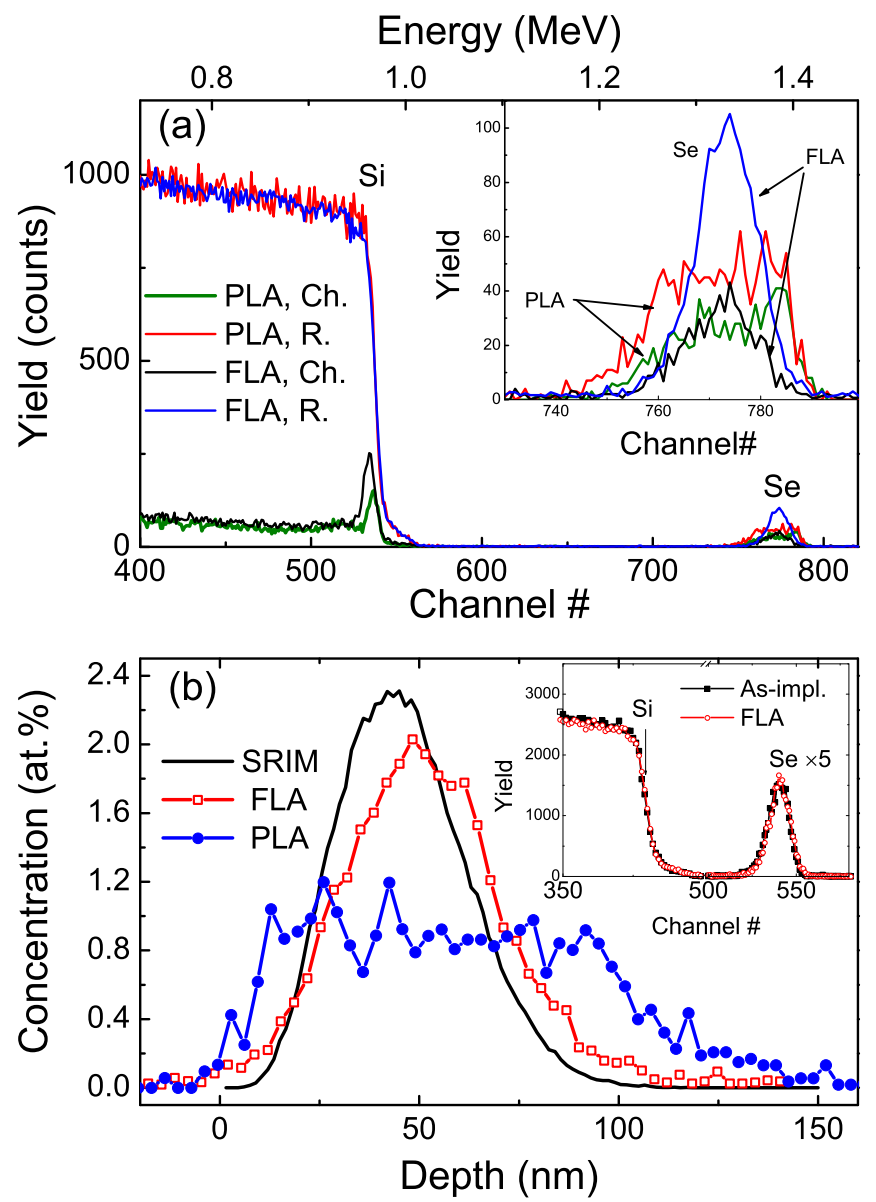

Figure $1 \mid$ (a) A sequence of $1.7 \mathrm{MeV}$ He RBS/channeling spectra of selenium implanted Si single crystals after different annealing (taking sample SiSe2.3 as the example). The channeling is along Si[001]. The Si matrix is recrystallized after both FLA $(1.3 \mathrm{~ms}, 3.4 \mathrm{kV})$ and PLA $(308 \mathrm{~nm}$, $28 \mathrm{~ns}, 0.9 \mathrm{~J} / \mathrm{cm}^{2}$ ). The inset shows a zoom for the selenium signal. We can see that selenium ions are mostly substitutional to the Si sites. However, for the PLA sample, selenium ions do not substitute Si sites at the near surface range. (b) The depth profile of selenium in Si after FLA or PLA calculated from RBS spectra. The projected range of selenium in FLA samples is in a reasonable agreement with SRIM simulation after considering the wellknown discrepancy in the projection range between SRIM simulation and the experimental values ${ }^{30-32}$. After PLA, a significant redistribution of selenium is observed. The inset shows the RBS spectra for the as-implanted and FLA samples measured using $1.5 \mathrm{MeV}$ He ions. There is no redistribution of selenium after FLA.

(pulse length: 1.3-20 millisecond). We also measured the random and channeling spectra of the unimplanted virgin substrate and the channeling spectrum for the as-implanted wafers (not shown to avoid overlapping). The implanted layer is fully amorphous. As shown in Fig. 1(a), the implanted layer can be well recrystallized by both annealing methods. The RBS-channeling spectrum reveals a near-surface minimum backscattered yield $\chi_{\text {min }}$ (the ratio of the aligned to random yields) of about $5 \%$, which is very close to the value of $4 \%$ determined for the virgin single crystal Si substrate.

In the selenium signal, two interesting features can be observed. One is the channeling effect, which occurs in both FLA and PLA samples. It proves the substitution of selenium onto the Si lattice sites, which is expected and has also been proved by other experiments ${ }^{20}$. The substitutional fraction can be approximated by $1-\chi_{\min }$ (selenium), which is shown in Fig. 2 for different samples. $\chi_{\text {min }}$ (selenium) stands for the ratio between the channeling and the random spectra for the selenium signal. As shown in Fig. 2(a) and (c), the selenium substitutional fraction is higher in the FLA sample than in the PLA sample. Another feature is the significant redistribution of selenium upon PLA. As shown in Fig. 1(b), selenium atoms move both inward and outward upon PLA, which has been reported in many papers ${ }^{2,20,26,27}$. However, there is no observable redistribution in the FLA sample as shown in the inset to Fig. 1(b). In RBS spectra, the selenium signal of the FLA sample almost overlaps with that of the as-implanted sample. The so-called snow plough effect $^{26,28}$ is avoided in solid phase epitaxy. In Fig. 1(b), we also show the depth distribution of selenium obtained by SRIM (Stopping and Range of Ions in Matter) simulation ${ }^{29}$. The slightly shallow and narrow distribution of selenium from simulation is well known $\mathrm{n}^{30,31}$ and is possibly due to the overestimation of electronic stopping for heavy ions in semiconductors ${ }^{32}$.

In Figure 2 we compare the selenium substitutional fraction for samples with different selenium concentration and with different annealing parameters. Figure 2(a) shows the substitutional fraction after FLA at different temperatures with the pulse duration of $1.3 \mathrm{~ms}$. The temperature is estimated according to calibration and theory model $^{33}$. All the samples are recrystallized. The substitutional fraction is between 40 and $70 \%$ and shows a maximum at the annealing temperature of $1473 \mathrm{~K}$. The substitutional fraction decreases with increasing the flash lamp pulse duration from $1.3 \mathrm{~ms}$ to $20 \mathrm{~ms}$ as shown in Fig. 2(b). This can be expected, since the diffusion length will be much larger if the sample is kept at higher temperature for longer time. For PLA samples, when the laser energy density is below $0.6 \mathrm{~J} / \mathrm{cm}^{2}$ the sample is not fully crystallized according to our RBS/ channeling measurements, particularly for the sample with smaller selenium concentration. The laser annealing parameters have been found to sensitively depend on the implantation fluence ${ }^{34,35}$. As shown in Fig. 2(c), when the pulsed laser annealing energy is $0.5 \mathrm{~J} /$ $\mathrm{cm}^{2}$, the substitutional fraction in sample SiSe1.1 is nearly zero. When the energy is high enough (larger than $0.6 \mathrm{~J} / \mathrm{cm}^{2}$ for both samples) to recrystallize the full layer, there is not much difference in the substitutional fraction. Yet the substitutional fraction for PLA samples is generally lower than for FLA samples. It is worthy to note that for both FLA or PLA samples, the substitutional fraction for sample SiSe1.1 is slightly smaller than for sample SiSe2.3, for which the reason is not clear and more investigation is required. The substitutional fraction has been estimated by comparing the integration of the selenium signal in channeling and random RBS spectra.

Table 1 | Sample definition and related parameters. The samples are referred as SiSe0.9PLA annealed by pulsed laser or SiSe0.9FLA annealed by flash lamp, respectively, with corresponding optimal parameters. The depth distribution of selenium (estimated thickness) is calculated using SRIM and verified by RBS measurements

\begin{tabular}{lccr} 
Sample ID & Implantation parameters & Estimated thickness & Selenium peak concentration (\%) \\
\hline SiSe0.9 & $110 \mathrm{keV}, 2.8 \times 10^{15} \mathrm{~cm}^{-2}$ & $\sim 150 \mathrm{~nm}$ & 0.9 \\
SiSe1.1 & $50 \mathrm{keV}, 1.4 \times 10^{15} \mathrm{~cm}^{-2}$ & & \\
$\mathrm{SiSe} 2.3$ & $60 \mathrm{keV}, 2.5 \times 10^{15} \mathrm{~cm}^{-2}$ & $\sim 100 \mathrm{~nm}$ & 1.1 \\
& $60 \mathrm{keV}, 5.0 \times 10^{15} \mathrm{~cm}^{-2}$ & $\sim 100 \mathrm{~nm}$ & 2.3
\end{tabular}




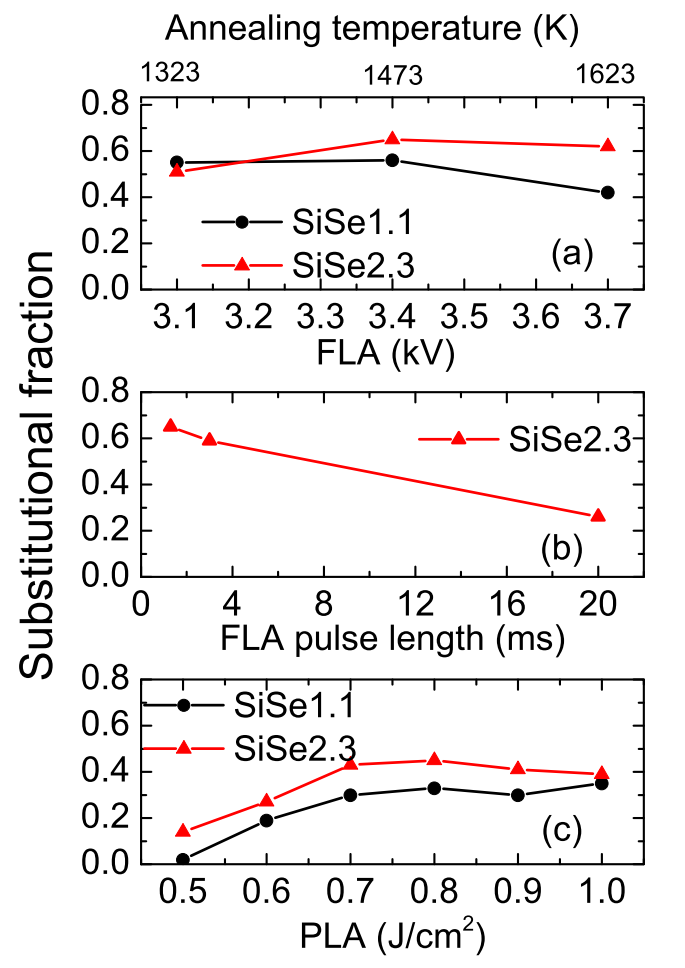

Figure $2 \mid$ The selenium substitutional fraction in selenium implanted Si after annealing by flash lamp or pulsed laser at different annealing energy: (a) FLA at different flash-lamp charging voltages (different annealing temperatures) with the pulse duration of $1.3 \mathrm{~ms}$, (b) FLA with different pulse duration and different charging voltages to have similar peak temperature $(\sim 1473 \mathrm{~K})$ in the sample and (c) PLA (308 nm, $28 \mathrm{~ns})$ with different energy densities.

Therefore, the relative error in the substitutional fraction values when comparing different samples is very small.

Structural properties: epitaxial recrystallization. The structural properties were characterized by Raman scattering and by X-ray diffraction (XRD). Figure 3(a) shows the Raman spectra obtained from selenium implanted $\mathrm{Si}$ as well as a reference single crystalline $\mathrm{Si}$. The as-implanted sample shows a broad Raman band at around $460 \mathrm{~cm}^{-1}$, which corresponds to the amorphous silicon formed during ion implantation ${ }^{36}$. After PLA or FLA, both samples exhibit a peak at $520 \mathrm{~cm}^{-1}$, corresponding to the transverse optical (TO) phonon mode of crystalline Si. Moreover, an additional peak at about $303 \mathrm{~cm}^{-1}$ which corresponds to the second-order transverseacoustic phonon (2TA) scattering from crystalline $\mathrm{Si}$ is observed for both samples as for the $\mathrm{Si}$ reference sample, which is a fingerprint of high crystallinity of the probed layer. The Raman results prove the complete recrystallization of selenium implanted Si by both PLA and FLA with relatively high quality.

As shown in Fig. 3(b), in the XRD $\theta-2 \theta$ scans the as-implanted sample shows a broad shoulder at the left side (lower angles). It is due to the crystalline damage induced by ion implantation. The implanted layer is amorphous-like as confirmed by Raman scattering (Fig. 3(a)). After annealing, the left-side shoulder disappears, indicating the recrystallization. There is large difference in the XRD patterns between the PLA and the FLA samples. When selenium ions substitute the Si sites, they form covalent bonds. The covalent radii for selenium and Si are 120 and $111 \mathrm{pm}$, respectively. The doping of selenium in the $\mathrm{Si}$ matrix up to a large enough concentration is expected to increase the lattice parameter, like in antimony doped $\mathrm{Si}^{37}$. We do observe this effect in the FLA sample. Note that the
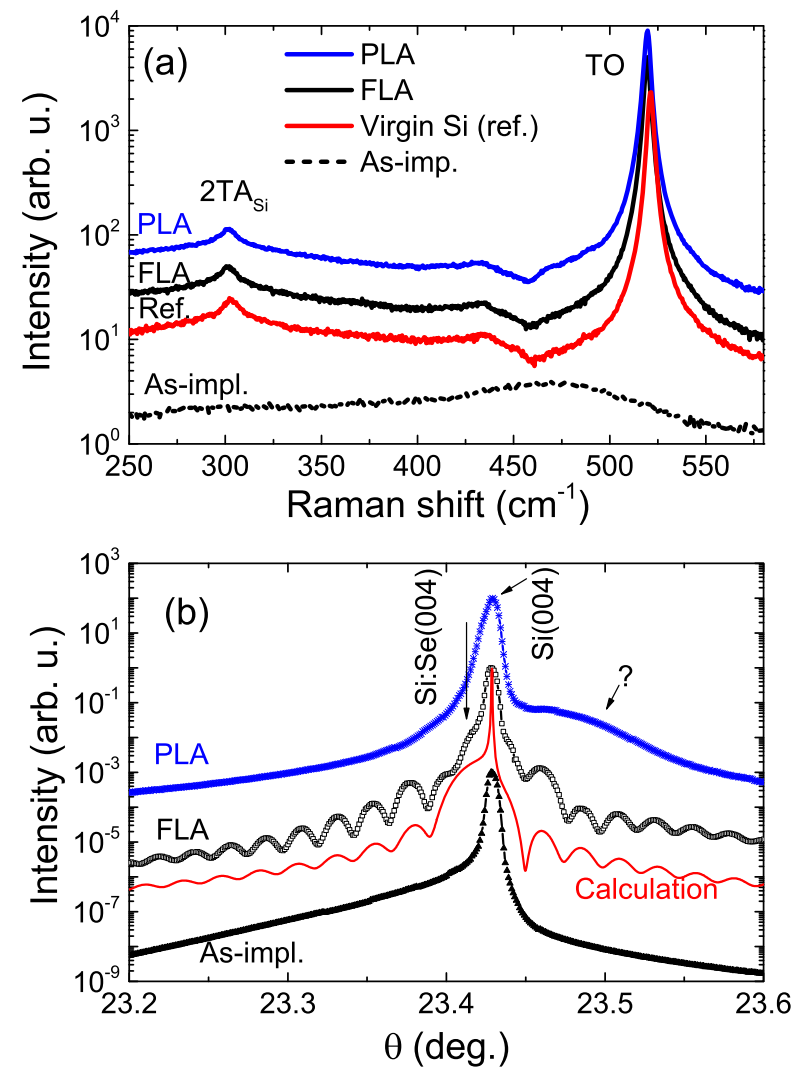

Figure $3 \mid$ Representative Raman or XRD results taking SiSe0.9 as the example after FLA $(1.3 \mathrm{~ms}, 3.4 \mathrm{kV})$ and PLA $\left(308 \mathrm{~nm}, 28 \mathrm{~ns}, 0.9 \mathrm{~J} / \mathrm{cm}^{2}\right)$. (a) $\mu$-Raman spectra: A virgin $\mathrm{Si}$ is also shown for comparison. The spectra have been vertically offset for clarity. (b) X-ray diffraction $\theta-2 \theta$ scans: The broad shoulder at the left side for the as-implanted sample is due to the ion beam induced damage. After annealing, this broad shoulder disappears and the oscillation indicates the good crystalline quality.

appearance of the $\mathrm{x}$-ray interference effect (Pendellösung) in the FLA sample is due to two layers with different lattice spacing. By fitting the XRD pattern, we obtain a top Si:Se layer with a lattice constant larger by around $0.60 \%$ compared with the Si substrate. The presence of the interference peaks also proves the high crystalline quality and the sharp interface after flashlamp annealing. However, the XRD result for the PLA sample is rather puzzling: it contains a broader shoulder at a larger diffraction angle corresponding to a smaller lattice constant.

Electrical properties: selenium hyperdoping. Selenium is a deep donor in Si and its energy level is around $200-300 \mathrm{meV}$ below the $\mathrm{Si}$ conduction band ${ }^{38}$. Upon high concentration doping, an insulator-metal transition was observed in selenium doped $\mathrm{Si}^{7}$. We also measured the electrical properties of selected samples. Figure 4 shows the sheet resistance in the temperature range 4$30 \mathrm{~K}$. Since we use nearly intrinsic $\mathrm{Si}$ substrate with a sheet resistance around $1.5 \times 10^{5} \mathrm{ohm} / \square$ at room temperature, the parallel resistance from the substrate is much larger than the selenium doped layer. Therefore, we only measure the conductivity from the doped Si layer. For the PLA samples, an insulator-metal transition occurs with increasing selenium concentration: Sample SiSe1.1 behaves like an insulator with its sheet resistance sharply rising at low temperature. Its conductivity is thermally activated. On the other hand, for the higher doped sample SiSe2.3 the resistance increases only very slightly at low temperature and its conductivity appears to remain finite when the temperature 


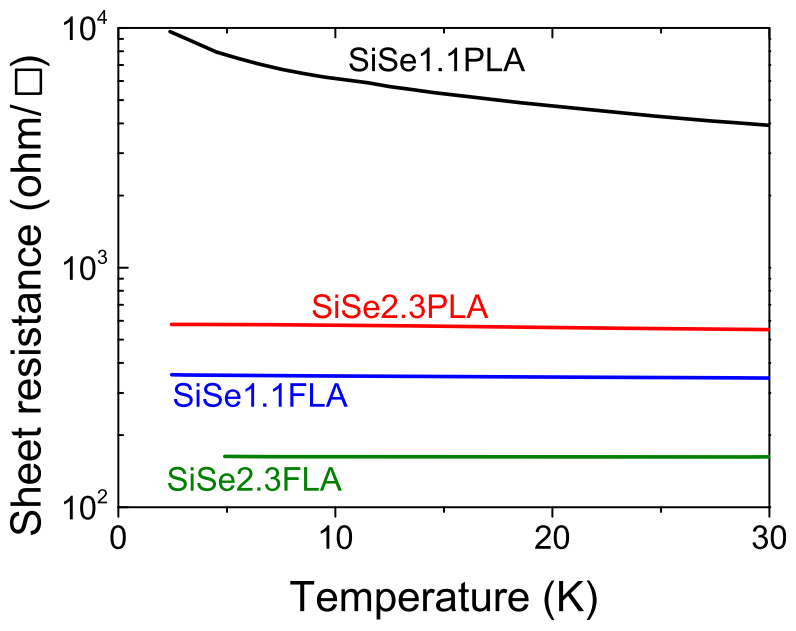

Figure 4 | Temperature dependent sheet resistance of selenium implanted Si annealed by FLA $(1.3 \mathrm{~ms}, 3.4 \mathrm{kV})$ or PLA $(308 \mathrm{~nm}, 28 \mathrm{~ns}$, $0.9 \mathrm{~J} / \mathrm{cm}^{2}$ ): With increasing selenium concentration, an insulator-metal transition occurs for the PLA samples, while all FLA samples show quasimetallic conduction.

approaches zero. In sharp contrast, flash lamp annealing renders both samples metallic like - the higher doped SiSe2.3, but also the lower doped SiSe1.1. The sheet resistance of sample SiSel.1FLA is even lower than SiSe2.3PLA with a higher Se concentration, clearly showing the superior (flash-lamp) annealing behavior by solidphase epitaxy. Finally sample SiSe2.3FLA exhibits the smallest sheet resistance and a clear metal-like conductivity. Its sheet resistance at $5 \mathrm{~K}$ is around $190 \mathrm{ohm} / \square$. It corresponds to a conductivity of $500 /(\mathrm{ohm} \cdot \mathrm{cm})$ if assuming a thickness of $100 \mathrm{~nm}$. This conductivity is three times larger than sample SiSe2.3PLA. We attribute the large conductivity to the high quality of the recrystallized layer by FLA, which results in a large Hall mobility.

Figure 5 shows the comparison of the electron concentration and Hall mobilities measured at $300 \mathrm{~K}$ for different FLA and PLA samples with increasing selenium concentration. The carrier concentration is calculated by assuming the effective thickness of the selenium doped layer as $150 \mathrm{~nm}$ for sample SiSe 0.9 and $100 \mathrm{~nm}$ for samples SiSe1.1 and SiSe2.3 (see Table 1), respectively. As can be seen, the carrier concentration is in the range of 3$13 \times 10^{19} \mathrm{~cm}^{-3}$. The activation efficiency is thus around $10 \%$. Although the PLA samples generally have a larger electron concentration than the FLA samples, the mobility is around 3 times smaller. The Hall mobility in FLA samples is in the range of $80-$ $100 \mathrm{~cm}^{2} / V \cdot s$. The values achieved here are almost of the same order as in Si doped with normal shallow donors in the high doping regime $\left(10^{19-20} \mathrm{~cm}^{-3}\right)^{39}$. It is worth to note that the FLA samples have a larger substitutional fraction and a smaller carrier concentration than that of PLA samples. This discrepancy might be due to the fact that in PLA samples selenium diffuses out- and in-ward resulting in a larger effective doping depth. On the other hand the interstitial selenium or other defects probably also contribute to free electrons as for the case of titanium supersaturated $\mathrm{Si}^{40,41}$.

\section{Discussion}

How to understand the success of hyperdoping Si with selenium via solid phase epitaxy? Let us revisit the mechanism for realizing hyperdoped semiconductors. The hyperdoping is attributed to the so-called solute trapping at the moving amorphous/crystalline interface when the dopant retain time is larger than the time required for one monolayer regrowth ${ }^{20,42,43}$. This process is determined by the diffusion coefficient, which is much larger in liquid

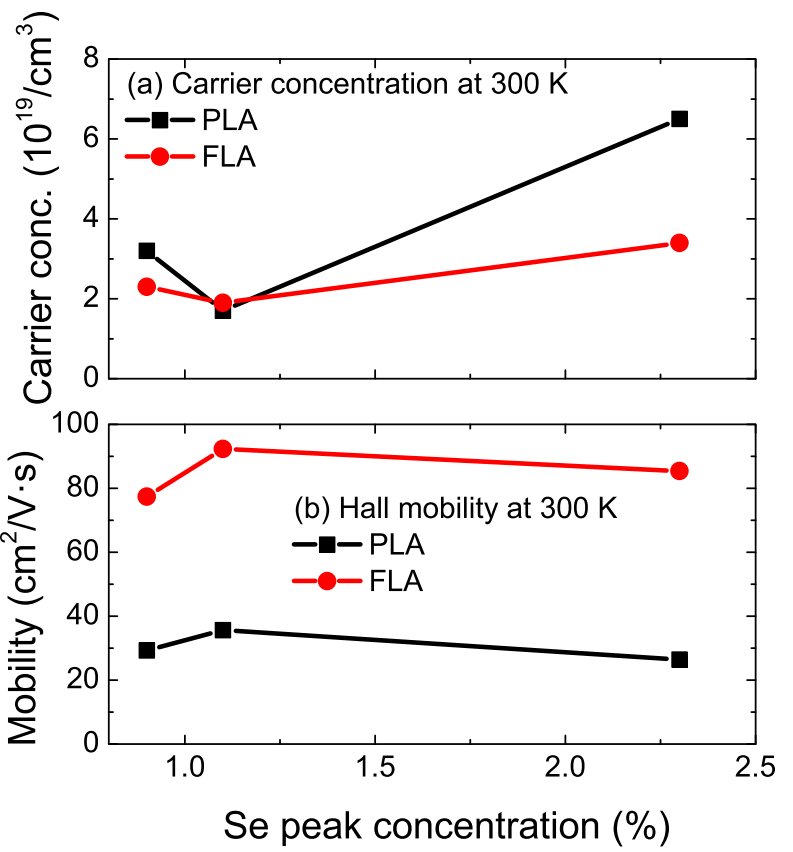

Figure 5 | (a) The carrier concentration and (b) the Hall mobility at $300 \mathrm{~K}$ of selenium implanted Si annealed by FLA $(1.3 \mathrm{~ms}, 3.4 \mathrm{kV})$ or PLA $\left(308 \mathrm{~nm}, 28 \mathrm{~ns}, 0.9 \mathrm{~J} / \mathrm{cm}^{2}\right)$ with optimized parameters. The mobility of FLA samples is comparable with the shallow donor considering the dopant concentration $^{39}$. The solid lines are only for guiding the eyes.

phase than in solid phase for most of dopants. This reminds us that a compromise approach might exist for chalcogen elements which are relatively slow diffusers in the solid phase compared with transition metals. Indeed, supersaturation of tellurium in $\mathrm{Si}$ up to $3 \times 10^{20} \mathrm{~cm}^{-3}$ has been realized by furnace annealing at $550^{\circ} \mathrm{C}$ and the substitutional fraction is around $70 \%$ in tellurium implanted $\mathrm{Si}^{20}$. However, the substitutional fraction is largely decreased to $45 \%$ when the implantation fluence is increased as for the case of selenium implanted $\mathrm{Si}^{44}$.

We try to characterize the competition between the solute trapping and diffusion by estimating the time needed to regrow $\left(\tau_{G}\right)$ or to diffuse $\left(\tau_{D}\right)$ over a Si monolayer $(0.27 \mathrm{~nm})$. In other words, the speed of the resolidification and the speed at which the impurity atoms can move determine how likely they will stay ahead or be trapped by the moving amorphous/crystalline interface. If $\tau_{D}$ is larger than $\tau_{G}$, the dopants are able to be trapped in the crystalline matrix. In Figure 6, we compare $\tau_{G}$ and $\tau_{D}$ estimated from data published in literature. $\tau_{D}$ is calculated according to the data in ref. 45. The large uncertainty in $\tau_{G}$ comes from the large scattering in the regrowth velocity, which exhibits different values reported by various groups $s^{20,46-49}$. However, as shown in Fig. 6, in solid phase Si $\tau_{D}$ is generally larger than $\tau_{G}$. That means selenium impurities can be trapped in the Si matrix if an optimized thermal treatment is applied even in solid phase processing. Particularly, in the low temperature regime, $\tau_{D}$ is much larger than $\tau_{G}$, which well explains the realization of doping above solubility limit by low temperature annealing ${ }^{20}$.

Another criterion to be considered is the annealing duration. In the regrown metastable layer, the impurity concentration is much above the thermal equilibrium solubility limit. During the prolonged annealing to finish the regrowth completely, the metastable solubility returns to the equilibrium value as dopants come out of their substitutional positions. This has been observed in sulfur hyperdoped $\mathrm{Si}^{50}$. The decrease in the substitutional fraction with increasing dop- 


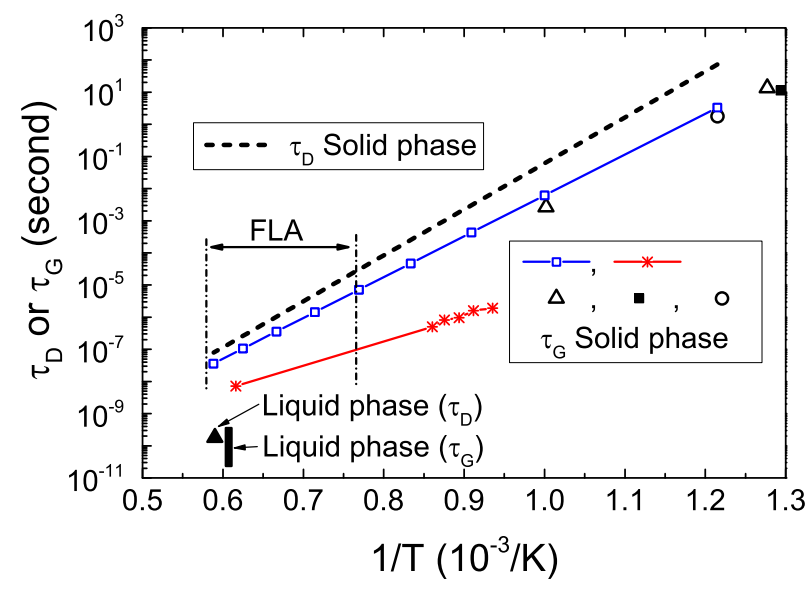

Figure 6 Competition between the Si recrystallization and selenium diffusion characterized by the time needed to regrow $\left(\tau_{G}\right)$ or diffuse $\left(\tau_{D}\right)$ one monolayer. The dashed line is $\tau_{D}$ in solid phase according to the diffusion parameter in Ref. 45 . The other lines or symbols are $\tau_{G}$ according to different references: open square ${ }^{46}, \operatorname{star}^{47}$, open triangle ${ }^{48}$, solid square ${ }^{49}$, open circle ${ }^{20}$. Despite the possible uncertainty of the regrowth velocity, $\tau_{G}$ is smaller than $\tau_{D}$. That means it is possible to trap selenium and realize metastable, selenium over-saturated Si layer. The vertical dashed and dotted lines indicate the working regime of flash lamp annealing (FLA). The reported $\tau_{G}$ is shown as a solid triangle ${ }^{2}$ and $\tau_{D}$ is shown as a vertical thick line by assuming the growth velocity of $1-10 \mathrm{~m} / \mathrm{s}$ for pulsed laser annealing (PLA).

ant concentration is due to incipient precipitation $^{51}$. For a first approximation, the metastable phase does not precipitate if the mean diffusion length of the impurity at the annealing temperature is less than their average distance. For a diffusion length larger than the average impurity distance, there is a finite probability of nucleation of a secondary phase or dimers. Therefore, the diffusion length $L=(2 D t)^{1 / 2}, \mathrm{D}$ being the diffusion coefficient ${ }^{45}$ and $t$ the annealing time, must be relatively short in order to achieve a high concentration of dopants on the substitutional sites. We illustrate the estimated results in Fig. 7, in which the working regimes of furnace annealing (FA), rapid thermal annealing (RTA) and flash lamp annealing (FLA) are indicated. In the case of FA at low temperature (around $500^{\circ} \mathrm{C}$ ), an annealing duration more than $1000 \mathrm{~s}$ is needed to regrow the whole layer. A longer annealing time and a slightly higher annealing temperature strongly increase the diffusion length. From Fig. 7, an annealing processing in millisecond or microsecond while keeping the system in solid phase could result in a minimized diffusion length. This also explains why the substitutional fraction strongly reduces in furnace annealed selenium-implanted samples when the selenium fluence is increased ${ }^{44}$.

In summary, we have presented a solid phase epitaxy approach by millisecond FLA to realize selenium hyperdoped Si from implanted amorphous layers. The dopant redistribution, which always occurs in pulsed-laser induced liquid phase epitaxy, can be effectively suppressed by FLA. The FLA-prepared sample exhibits larger Hall mobility and conductivity than the samples prepared by liquid phase epitaxy. The success of FLA lies in the facts that the selenium impurities have moderate diffusion coefficients in solid Si and that FLA occurs within milliseconds (much shorter than furnace or rapid thermal annealing). The regrowth velocity can beat the dopant diffusion in carefully optimized annealing condition. Our finding is not limited to selenium, but generally interesting for chalcogen impurities and other unconventional dopants in semiconductors. Annealing in the microsecond or millisecond range might be optimized for realizing hyperdoping of transition metal impurities in $\mathrm{Si}$ as well as in III-V compound semiconductors.

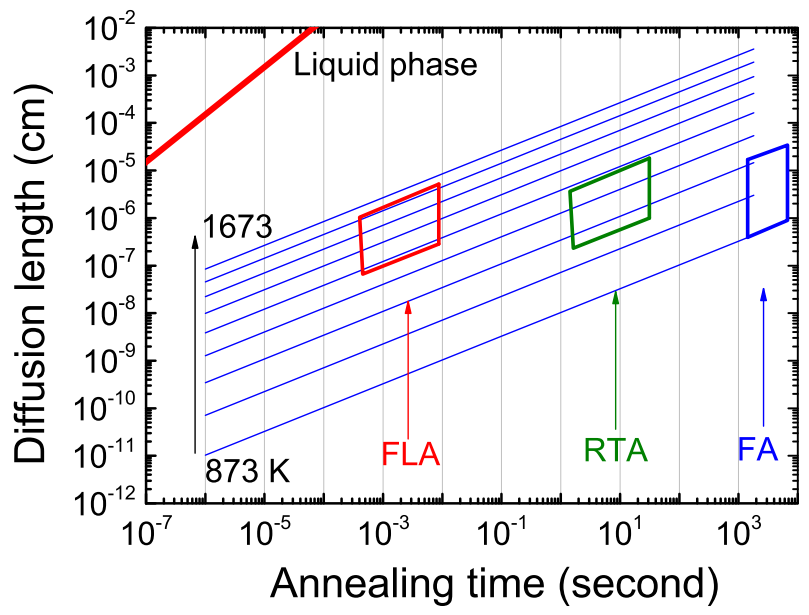

Figure 7 Selenium diffusion length $(L)$ in Si at different temperature vs. time duration. The three boxes indicate the working regime of different thermal process in solid phase: low temperature furnace annealing (FA) for more than 1000 second, rapid thermal annealing (RTA) for seconds and flash lamp annealing (FLA) for milliseconds at high temperature. The diffusion length of selenium in liquid phase is also shown for comparison.

\section{Methods}

Semi-insulating (100) Si wafers were implanted with selenium at room temperature The implantation energy and fluence are listed in table $\mathrm{I}$. The resistivity of the virgin $\mathrm{Si}$ wafer is above $4000 \mathrm{ohm} \cdot \mathrm{cm}$ at room temperature. This resistivity corresponds to a sheet resistance of $1.5 \times 10^{4} \Omega / \square$ at room temperature, which is even 10 times larger than sample SiSe1.1PLA (shown in Fig. 4) with the most resistive sample in our experiment. Therefore, the parallel conductivity from the Si substrate can be neglected.

Implanted Si samples were flash-lamp annealed for $1.3,3$ and $20 \mathrm{~ms}$. The FLA system employed in our experiments has been introduced in details in Ref. 33,52. It consists of two annealing systems. At the top there are twelve $30 \mathrm{~cm}$ long xenon (Xe) lamps spaced by about $3 \mathrm{~cm}$ representing together with the reflector the FLA system, and at the bottom a lower bank of halogen lamps allows the wafer to be preheated to a selected temperature - a type of rapid thermal annealing system. The Xe lamps are energized by discharging a capacitor/inductor unit in the millisecond time scale. The maximum energy which can be delivered to the sample during a single $20 \mathrm{~ms}$ shot is $250 \mathrm{~kJ}$ which is sufficient to melt silicon. The emission spectrum of Xe lamps used in our system falls in the visible spectral range in between 350 and $800 \mathrm{~nm}$. For effective annealing, FLA treated materials should have a high absorption coefficient in this spectral region, e.g. silicon is perfectly suited for FLA processes. Wafers up to $100 \mathrm{~mm}$ in diameter can be processed with a lateral homogeneity better than $5 \%$. The temperature simulation was carried out based on the wave transfer matrix method for modelling the absorption of the flash light, and the numerical solution of the one-dimensional heat equation ${ }^{53}$. The optical system taken into consideration for temperature simulation comprises the inert gas atmosphere of argon, amorphous Si layer formed during ion implantation and the bulk Si. The energy delivered to the sample during the FLA process depends on the overall absorption and transmission of the investigated system. The as-implanted $\mathrm{Si}$ absorb about $85 \%$ of the incident flash lamp spectrum, while the virgin $\mathrm{Si}$ wafer absorb only about $65 \%$ of the light delivered to the sample surface. Therefore the annealing parameters used to obtain the same final temperature during the FLA process in different materials have to be carefully adjusted. As one example, according to the simulation of the temperature distribution in Si wafer during $3 \mathrm{~ms}$ annealing, the maximum temperature at the surface is reached after $2.5 \mathrm{~ms}$ with temperature gradient of about $1^{\circ} \mathrm{C} / \mu \mathrm{m}$.

The laser annealing system is a Coherent $\mathrm{XeCl}$ excimer laser with $308 \mathrm{~nm}$ wavelength and $28 \mathrm{~ns}$ duration which was focused to a $5 \mathrm{~mm} \times 5 \mathrm{~mm}$ square spot, where the lateral intensity variation was less than $3 \%$. Samples were irradiated with different energy fluences ranging from $0.5 \mathrm{~J} / \mathrm{cm}^{2}$ to $1.2 \mathrm{~J} / \mathrm{cm}^{2}$

The structural properties of the annealed samples were investigated by Raman spectroscopy, Rutherford backscattering spectrometry/channeling (RBS/channeling) and X-ray diffraction (XRD). The RBS measurements were performed with a collimated $1.7 \mathrm{MeV} \mathrm{He}^{+}$beam at a backscattering angle of $170^{\circ}$. For the results in the inset of Fig. 1(b), the measurements were performed with $1.5 \mathrm{MeV} \mathrm{He}^{+}$ions. The sample was mounted on a three-axis goniometer with a precision of $0.01^{\circ}$. The channeling spectra were measured by aligning the sample to make the impinging $\mathrm{He}^{+}$beam parallel with the Si[001] axis. The XRD experiment were performed at Rossendorf Beamline (ROBL), European Synchrotron Radiation Facility (ESRF), using a X-ray with wavelength of $0.1078 \mathrm{~nm}$. The phonon modes were determined by Raman spectroscopy in a backscattering geometry in the range of 200 to $600 \mathrm{~cm}^{-1}$ using a $532 \mathrm{~nm} \mathrm{Nd:YAG} \mathrm{laser} \mathrm{with} \mathrm{a} \mathrm{liquid} \mathrm{nitrogen} \mathrm{cooled} \mathrm{charge} \mathrm{coupled} \mathrm{device}$ camera. Electrical properties were measured in van der Pauw geometry using a 
commercial Lakeshore Hall System. Gold electrodes were sputtered onto the four corners of the square-like samples. The sputtering process also removed the nature $\mathrm{SiO}_{2}$ layer to some extent. Silver glue was used to contact the wires to the gold electrodes. All contacts are confirmed to be ohmic as we checked by measuring current-voltage curves at different temperatures.

1. Kim, T. G., Warrender, J. M. \& Aziz, M. J. Strong sub-band-gap infrared absorption in silicon supersaturated with sulfur. Appl. Phys. Lett 88, 241902; DOI:10.1063/1.2212051 (2006)

2. Bob, B. P. et al. Fabrication and subband gap optical properties of silicon supersaturated with chalcogens by ion implantation and pulsed laser melting. J. Appl. Phys 107, 123506; DOI:10.1063/1.3415544 (2010).

3. Tabbal, M., Kim, T., Woolf, D., Shin, B. \& Aziz, M. Fabrication and sub-band-gap absorption of single-crystal Si supersaturated with Se by pulsed laser mixing. Appl. Phys. A 98, 589-594; DOI:10.1007/s00339-009-5462-1 (2010).

4. Umezu, I. et al. Emergence of very broad infrared absorption band by hyperdoping of silicon with chalcogens. J. Appl. Phys 113, 213501; DOI:10.1063/ 1.4804935 (2013).

5. Hu, S. et al. Structural and optoelectronic properties of selenium-doped silicon formed using picosecond pulsed laser mixing. Phys. Status Solidi (a) 209, 2521-2526; DOI:10.1002/pssa.201228202 (2012).

6. Winkler, M. T. et al. Insulator-to-Metal Transition in Sulfur-Doped Silicon. Phys. Rev. Lett 106, 178701; DOI:10.1103/PhysRevLett.106.178701 (2011).

7. Ertekin, E. et al. Insulator-to-Metal Transition in Selenium-Hyperdoped Silicon: Observation and Origin. Phys. Rev. Lett 108, 026401; DOI:10.1103/ PhysRevLett.108.026401 (2012)

8. Carey, J. E., Crouch, C. H., Shen, M. \& Mazur, E. Visible and near-infrared responsivity of femtosecond-laser microstructured silicon photodiodes. Opt. Lett 30, 1773-1775; DOI:10.1364/OL.30.001773 (2005).

9. Said, A. J. et al. Extended infrared photoresponse and gain in chalcogensupersaturated silicon photodiodes. Appl. Phys. Lett 99, 073503; DOI:10.1063/ 1.3609871 (2011).

10. Hu, S. et al. Improved photoresponse characteristics in Se-doped Si photodiodes fabricated using picosecond pulsed laser mixing. Semicond. Sci. Technol 27, 102002; DOI:10.1088/0268-1242/27/10/102002 (2012).

11. Luque, A. \& Martí, A. Increasing the Efficiency of Ideal Solar Cells by Photon Induced Transitions at Intermediate Levels. Phys. Rev. Lett 78, 5014-5017; DOI:10.1103/PhysRevLett.78.5014 (1997).

12. Antolín, E. et al. Lifetime recovery in ultrahighly titanium-doped silicon for the implementation of an intermediate band material. Appl. Phys. Lett 94, 042115; DOI:10.1063/1.3077202 (2009).

13. Sánchez, K., Aguilera, I., Palacios, P. \& Wahnón, P. Formation of a reliable intermediate band in Si heavily coimplanted with chalcogens ( $\mathrm{S}, \mathrm{Se}, \mathrm{Te}$ ) and group III elements (B, Al). Phys. Rev. B 82, 165201; DOI:10.1103/PhysRevB.82.165201 (2010).

14. Guenther, K.-M. et al. Excess carrier generation in femtosecond-laser processed sulfur doped silicon by means of sub-bandgap illumination. Appl. Phys. Lett 104, 042107; DOI:10.1063/1.4863439 (2014).

15. Carlson, R., Hall, R. \& Pell, E. Sulfur in silicon. J. Phys. Chem. Solids 8, 81-83; DOI:10.1016/0022-3697(59)90279-3 (1959).

16. Sheehy, M. A., Tull, B. R., Friend, C. M. \& Mazur, E. Chalcogen doping of silicon via intense femtosecond-laser irradiation. Mater. Sci. \& Eng. B 137, 289-294; DOI:10.1016/j.mseb.2006.10.002 (2007)

17. Smith, M. J. et al. Improving dopant incorporation during femtosecond-laser doping of Si with a Se thin-film dopant precursor. Appl. Phys. A 114, 1009-1016; DOI:10.1007/s00339-013-7673-8 (2014).

18. Tabbal, M. et al. Formation of single crystal sulfur supersaturated silicon based junctions by pulsed laser melting. J. Vac. Sci. Technol. B 25, 1847-1852; DOI:10.1116/1.2796184 (2007)

19. Stritzker, B., Pospieszczyk, A. \& Tagle, J. Measurement of lattice temperature of silicon during pulsed laser annealing. Phys. Rev. Lett 47, 356; DOI:10.1103/ PhysRevLett.47.356 (1981).

20. Campisano, S. U., Foti, G., Baeri, P., Grimaldi, M. G. \& Rimini, E. Solute trapping by moving interface in ionimplanted silicon. Appl. Phys. Lett 37, 719-722; DOI:10.1063/1.92057 (1980)

21. Aziz, M. Model for solute redistribution during rapid solidification. J. Appl. Phys 53, 1158-1168; DOI:10.1063/1.329867 (1982).

22. Aziz, M., Tsao, J., Thompson, M. O., Peercy, P. \& White, C. Solute trapping: comparison of theory with experiment. Phys. Rev. Lett 56, 2489; DOI:10.1103/ PhysRevLett.56.2489 (1986).

23. Aziz, M. J. \& Kaplan, T. Continuous growth model for interface motion during alloy solidification. Acta metallurgica 36, 2335-2347; DOI:10.1016/00016160(88)90333-1 (1988).

24. Celler, G., Poate, J. \& Kimerling, L. controlled crystal regrowth of ion-implanted silicon by laser irradiation. Appl. Phys. Lett 32, 464-466; DOI:10.1063/1.90109 (1978).

25. Dubon, O., Scarpulla, M., Farshchi, R. \& Yu, K. Doping and defect control of ferromagnetic semiconductors formed by ion implantation and pulsed-laser melting. Physica B 376, 630-634; DOI:10.1016/j.physb.2005.12.159 (2006).

26. Baeri, P., Campisano, S. U., Foti, G. \& Rimini, E. Segregation Effects in CuImplanted Si after Laser-Pulse Melting. Phys. Rev. Lett 41, 1246-1249; DOI:10.1103/PhysRevLett.41.1246 (1978).
27. Recht, D. et al. Supersaturating silicon with transition metals by ion implantation and pulsed laser melting. J. Appl. Phys 114, 124903; DOI:10.1063/1.4821240 (2013).

28. Baeri, P. et al. Dependence of trapping and segregation of indium in silicon on the velocity of the liquid-solid interface. Appl. Phys. Lett 37, 912-914; DOI:10.1063/ 1.91856 (1980)

29. Ziegler, J. F., Ziegler, M. D. \& Biersack, J. P. SRIM-The stopping and range of ions in matter (2010). Nucl. Inst. \& Meth. in Phys. Res. B 268, 1818-1823; DOI:10.1016/ j.nimb.2010.02.091 (2010).

30. Fichtner, P. et al. Range profiles of 10 to $390 \mathrm{keV}$ ions $(29<\mathrm{Z}<83)$ implanted into amorphous silicon. Nucl. Inst. \& Meth. in Phys. Res. B 28, 481-487; DOI:10.1016/ 0168-583X(87)90491-5 (1987)

31. Palmetshofer, L., Gritsch, M. \& Hobler, G. Range of ion-implanted rare earth elements in $\mathrm{Si}$ and $\mathrm{SiO}_{2}$. Mater. Sci. \& Eng. B 81, 83-85; DOI:10.1016/S09215107(00)00742-X (2001).

32. Jin, K., Zhang, Y., Xue, H., Zhu, Z. \& Weber, W. J. Ion distribution and electronic stopping power for Au ions in silicon carbide. Nucl. Inst. \& Meth. in Phys. Res. B 307, 65-70; DOI:10.1016/j.nimb.2013.02.051 (2013).

33. Skorupa, W. et al. Advanced thermal processing of ultrashallow implanted junctions using flash lamp annealing. J. Electrochem. Soc 152, G436-G440; DOI:10.1149/1.1899268 (2005).

34. Venkatesan, T., Golovchenko, J., Poate, J., Cowan, P. \& Celler, G. Dose dependence in the laser annealing of arsenic-implanted silicon. Appl. Phys. Lett 33, 429-431; DOI:10.1063/1.90411 (1978)

35. Bean, J. et al. Substrate and doping effects upon laser-induced epitaxy of amorphous silicon. J. Appl. Phys 50, 881-885; DOI:10.1063/1.326004 (1979).

36. Prucnal, S. et al. Formation of inas quantum dots in silicon by sequential ion implantation and flash lamp annealing. Appl. Phys B 101, 315-319; DOI:10.1007/ s00340-010-4140-5 (2010).

37. Radamson, H., Sardela Jr, M., Hultman, L. \& Hansson, G. Characterization of highly Sb-doped Si using high-resolution $\mathrm{x}$-ray diffraction and transmission electron microscopy. J. Appl. Phys 76, 763-767; DOI:10.1063/1.357778 (1994).

38. Pantelides, S. T. Deep centers in semiconductors (CRC Press, 1992).

39. Jacoboni, C., Canali, C., Ottaviani, G. \& Alberigi Quaranta, A. A review of some charge transport properties of silicon. Solid-State Electron 20, 77-89; DOI:10.1016/0038-1101(77)90054-5 (1977).

40. Sánchez, K., Aguilera, I., Palacios, P. \& Wahnón, P. Assessment through firstprinciples calculations of an intermediate-band photovoltaic material based on Ti-implanted silicon: Interstitial versus substitutional origin. Phys. Rev. B 79, 165203; DOI:10.1103/PhysRevB.79.165203 (2009).

41. Pastor, D. et al. Interstitial Yi for intermediate band formation in $\mathrm{Ti}$ supersaturated silicon. J. Appl. Phys 112, 113514; DOI:10.1063/1.4768274 (2012).

42. Duffy, R. et al. Impurity redistribution due to recrystallization of preamorphized silicon. J. Vac. Sci. Technol. B 23, 2021-2029; DOI:10.1116/1.2044813 (2005).

43. Wündisch, C. et al. Millisecond flash lamp annealing of shallow implanted layers in Ge. Appl. Phys. Lett 95, 252107; DOI:10.1063/1.3276770 (2009).

44. Mao, X. et al. Optical and Electrical Properties of Single-Crystal Si Supersaturated with Se by Ion Implantation. Chin. Phys. Lett 29, 097101; DOI:10.1088/0256307X/29/9/097101 (2012)

45. Vydyanath, H. R., Lorenzo, J. S. \& Kroeger, F. A. Defect pairing diffusion, and solubility studies in selenium-doped silicon. J. Appl. Phys 49, 5928-5937; DOI:10.1063/1.324560 (1978)

46. Kokorowski, S. A., Olson, G. L. \& Hess, L. D. Kinetics of laserinduced solid phase epitaxy in amorphous silicon films. J. Appl. Phys 53, 921-926; DOI:10.1063/ 1.330561 (1982).

47. Lietoila, A., Gold, R. \& Gibbons, J. The rate of $\mathrm{cw}$ laser induced solid phase epitaxial regrowth of amorphous silicon. Appl. Phys. Lett 39, 810-812; DOI:10.1063/1.92566 (1981).

48. Roth, J. A., Olson, G. L., Jacobson, D. C. \& Poate, J. M. Kinetics of solid phase epitaxy in thick amorphous Si layers formed by $\mathrm{MeV}$ ion implantation. Appl. Phys. Lett 57, 1340-1342; DOI:10.1063/1.103477 (1990).

49. Csepregi, L., Kennedy, E., Mayer, J. \& Sigmon, T. Substrate-orientation dependence of the epitaxial regrowth rate from Si-implanted amorphous Si. J. Appl. Phys 49, 3906-3911; DOI:10.1063/1.325397 (1978).

50. Simmons, C. et al. Deactivation of metastable single-crystal silicon hyperdoped with sulfur. J. Appl. Phys 114, 243514; DOI:10.1063/1.4854835 (2013).

51. Campisano, S. U., Rimini, E., Baeri, P. \& Foti, G. Supersaturated solid solutions after solid phase epitaxial growth in Biimplanted silicon. Appl. Phys. Lett 37, 170-172; DOI:10.1063/1.91812 (1980).

52. McMahon, R. et al. Flash-lamp annealing of semiconductor materialsapplications and process models. Vacuum 81, 1301-1305; DOI:10.1016/j.vacuum.2007.01.033 (2007).

53. Smith, M., McMahon, R., Voelskow, M. \& Skorupa, W. Modeling and regrowth mechanisms of flash lamp processing of SiC-on-silicon heterostructures. J. Appl. Phys 96, 4843-4851; DOI:10.1063/1.1786650 (2004).

\section{Acknowledgments}

We would like to thank the ion implanter group at HZDR for technical support and Olga Roshchupkina for her assistance with synchrotron XRD. The Helmholtz-Association (HGF-VH-NG-713) is gratefully acknowledged. The author (FL) thanks the support by 
China Scholarship Council (File No. 201307040037). The discussion with Karl-Heinz Heinig is greatly acknowledged.

\section{Author contributions}

S.Z. designed the research and wrote the manuscript. F.L. did the pulsed laser annealing and RBS measurements. S.P. performed flashlamp annealing. K.G. and M.K. carried out the Raman and transport measurements, respectively. C.B. contributed to the XRD measurement and data analysis. M.P. contributed to the thermal dynamic estimation. W.S. and M.H. supervised the work. All authors contributed to discussion and correction for the manuscript.

\section{Additional information}

Competing financial interests: The authors declare no competing financial interests.

How to cite this article: Zhou, S. et al. Hyperdoping silicon with selenium: solid vs. liquid phase epitaxy. Sci. Rep. 5, 8329; DOI:10.1038/srep08329 (2015).

(c) (i)

This work is licensed under a Creative Commons Attribution 4.0 International License. The images or other third party material in this article are included in the article's Creative Commons license, unless indicated otherwise in the credit line; if the material is not included under the Creative Commons license, users will need to obtain permission from the license holder in order to reproduce the material. To view a copy of this license, visit http://creativecommons.org/licenses/by/4.0/ 